\title{
Moderate Intensity Resistance Training Significantly Elevates Testosterone following Upper Body and Lower Body Bouts When Total Volume is Held Constant
}

\author{
Robert Rietjens \\ Department of Kinesiology and Nutrition Sciences, University of Nevada, Las Vegas \\ 4505 S. Maryland Parkway, Box 453034, Las Vegas, NV, USA \\ Tori M. Stone \\ Department of Kinesiology and Nutrition Sciences, University of Nevada, Las Vegas \\ 4505 S. Maryland Parkway, Box 453034, Las Vegas, NV, USA \\ Jeffrey Montes \\ Department of Kinesiology and Nutrition Sciences, University of Nevada, Las Vegas \\ 4505 S. Maryland Parkway, Box 453034, Las Vegas, NV, USA \\ John C. Young \\ Department of Kinesiology and Nutrition Sciences, University of Nevada, Las Vegas \\ 4505 S. Maryland Parkway, Box 453034, Las Vegas, NV, USA \\ E-mail: jack.young@unlv.edu \\ Richard D. Tandy \\ Department of Kinesiology and Nutrition Sciences, University of Nevada, Las Vegas \\ 4505 S. Maryland Parkway, Box 453034, Las Vegas, NV, USA \\ E-mail: dick.tandy@unlv.edu \\ Jenifer C. Utz \\ School of Life Sciences, University of Nevada, Las Vegas \\ 4505 S. Maryland Parkway, Box 454004, Las Vegas, NV, USA \\ E-mail: jenifer.utz@unlv.edu \\ James W. Navalta (Corresponding author) \\ Department of Kinesiology and Nutrition Sciences, University of Nevada, Las Vegas \\ 4505 S. Maryland Parkway, Box 453034, Las Vegas, NV, USA \\ E-mail: james.navalta@unlv.edu
}

Received: 11-09- 2015

Accepted: 18-10- 2015

Published: 31-10- 2015

doi:10.7575/aiac.ijkss.v.3n.4p.50

URL: http://dx.doi.org/10.7575/aiac.ijkss.v.3n.4p.50

\begin{abstract}
Introduction: It is unknown whether resistance training intensity or total volume of work affects the acute testosterone response to a greater extent. Purpose: Therefore, the circulating testosterone response was investigated following four resistance training protocols where total volume of work was held constant: moderate intensity (70\% 1RM) upper body (bench press, bent barbell row, and military press), moderate intensity lower body (squat and deadlift), high intensity (90\% 1RM) upper body, high intensity lower body. Methods: Total volume of work performed by each participant between protocols was maintained by adjusting the number of sets and or repetitions performed. Ten healthy, resistance trained men volunteered, and performed exercise protocols on separate days in a counterbalanced order. Capillary blood was obtained via finger stick at baseline (pre), immediately following the exercise session (post), and $1 \mathrm{~h}$ post for the determination of testosterone concentration. Data were analyzed using a factorial ANOVA and significance was accepted at $\mathrm{p} \leq 0.05$. Results: Both moderate intensity resistance protocols (upper and lower body) significantly increased testosterone concentration ( $\mathrm{p}=0.026$, and $\mathrm{p}=0.024$ respectively), whereas the high intensity protocols elevated testosterone but failed to achieve significance (upper $\mathrm{p}=0.272$, lower $\mathrm{p}=0.658$ ). No difference was noted in post session testosterone concentration between upper and lower body protocols for either moderate $(\mathrm{p}=0.248)$ or high intensity $(\mathrm{p}=0.990)$. Conclusion: This may be useful for novice resistance trained individuals because it provides evidence that moderate intensity is sufficient to increase testosterone compared to high intensity protocols that could be associated with a greater risk of injury.
\end{abstract}

Keywords: hormone response, equal total work, high intensity protocol 


\section{Introduction}

Acute bouts of heavy resistance exercise induce a myriad of physiological responses. These acute responses can lead to adaptations over time that are necessary for increasing muscular strength and hypertrophy. Hormonal responses to resistance training are among the most important for increasing size and strength of the musculature exposed to a significant stimulus (Vingren et al., 2010). Increased anabolic hormone concentration after an acute bout of resistance exercise can lead to increased protein synthesis within the muscle being trained (Kraemer \& Ratamess, 2005). It is important to note that the larger the stimulus the greater the endocrine response expected. A larger training volume or higher training intensity should lead to an increased endocrine response. Ultimately, manipulation of these training variables can lead to tissue remodeling via increased protein synthesis due to the endocrine response following a bout of heavy resistance exercise.

One of the major anabolic hormones associated with increased muscle size and strength is testosterone. Testosterone's primary biological effects are nitrogen retention, stimulation of protein synthesis, and development of secondary sex characteristics such as increased hair growth (Vingren et al., 2010). Testosterone production begins in the Leydig cells located in the testes of men. It is also produced, to a smaller degree, in the ovaries and adrenal cortex in women. The central nervous system stimulates the hypothalamus to begin testosterone production, which suggests a strong link between the nervous and endocrine systems (Vingren et al., 2010). Fahey and colleagues demonstrated that testosterone increases immediately post-training after performing 3 sets of 5 repetitions maximum for the bench press, seated press, and leg press (Fahey, Rolph, Moungmee, Nagel, \& Mortara, 1976). This provides evidence that heavy resistance training of five repetitions or less with near maximal loads, stimulates a significant release in testosterone following a training bout. This also suggests that the larger the muscle mass that is activated the larger the response will be. Exercises such as bench press, leg press, and latissimus dorsi pulldowns have been shown to increase testosterone post exercise to a greater extent than small muscle mass movements when performed above $80 \%$ of one repetition maximum (Weiss, Cureton, \& Thompson, 1983). This demonstrates as exercise intensity, or a given load or resistance for a certain exercise increases, testosterone release will increase proportionally following the bout.

Upper body training exclusively has not been shown to be a strong enough stimulus in order to elicit a significant post-exercise hormonal response (Migiano et al., 2010). Even three sets of 10 repetitions at $80 \%$ of 1 repetition maximum for five different upper body exercises was not enough to observe a significant elevation in testosterone post-exercise (Migiano et al., 2010). Lower body training, however, has been shown to induce a rise in testosterone immediately post-exercise even when only one exercise is performed. When 8 sets of 10 repetitions for the back squat exercise was performed at 10 repetition maximum, an elevation in testosterone immediately post-exercise was observed (Hough, Papacosta, Wraith, \& Gleeson, 2011).

The amount of total work in Joules $(\mathrm{J})$ has been shown to directly relate to the concentration of testosterone measured post-exercise (Hakkinen, Pakarinen, Newton, \& Kraemer, 1998). Gotshalk et al. demonstrated that a protocol consisting of 3 sets of 10 repetitions was superior compared with 1 set of 10 repetitions in regards to the amount of testosterone release post-exercise (Gotshalk et al., 1997). The American College of Sports Medicine recommends that an individual complete one set of anywhere from 3 to 20 repetitions for every major muscle group to improve muscular strength (Pescatello et al., 2014). This is in contrast to many exercise programs that require multiple sets of a higher intensity. It is unknown what the testosterone response will be for protocols of different intensity that have the same amount of total work in Joules. The primary purpose of this study was to observe if there was a different testosterone response between protocols of varying intensity but that had the same amount of total work ( 2 sets of 9 repetitions at $70 \% 1$ repetition maximum [RM] versus 5 sets of 3 repetitions at $90 \% 1 \mathrm{RM}$ ). These protocols maintained the same amount of total work (J) by adjusting the set, repetition, and intensity schemes. A secondary objective was to observe a difference in the endocrine response to upper versus lower body training while total training volume remained constant. We hypothesized that there would be a significantly higher testosterone response immediately post-workout for both the moderate and high intensity groups for both upper and lower body. Our second hypothesis was that lower body training would induce a significantly greater testosterone concentration immediately post-workout when compared to upper body training.

\section{Methods}

\subsection{Subjects}

Using the data from Gotshalk et al. for the pre to post difference in testosterone when three sets were completed, an effect size of 0.7035 was calculated (Gotshalk et al., 1997). Utilizing G*Power software, a power analysis revealed that a total of nine participants would be sufficient to detect significant effects if present (Faul, Erdfelder, Lang, \& Buchner, 2007). To take a conservative approach, ten recreationally active, apparently healthy males were recruited to participate in this study (see Table 1). Subjects were screened prior to participation according to the ACSM health risk algorithm, and only individuals who were considered "low risk" completed the exercise intervention. Recreationally trained was defined as individuals who participated in regular weight training at least twice per week for the past six months. Investigation protocols were approved by the institutional review board (protocol \# 1311 4642), and participants provided signed informed consent prior to participation. 
Table 1. Participant characteristics who completed acute upper and lower body resistance training exercises at $70 \%$ and $90 \%$ of 1 -repetition maximum

\begin{tabular}{lcccc}
\hline & Age $(\mathrm{yrs})$ & Height $(\mathrm{cm})$ & Weight $(\mathrm{kg})$ & Body fat $(\%)$ \\
\hline Mean & 22.80 & 176.78 & 79.73 & 16.39 \\
Standard Deviation & 1.545 & 11.12 & 11.45 & 4.46 \\
\hline
\end{tabular}

\subsection{Procedures}

Prior to the visit, participants were instructed to wear appropriate athletic attire, be adequately hydrated, and not consume anything with calories at least 3 hours before testing. The participants were also instructed to not consume any alcohol 24 hours prior to testing and to refrain from vigorous exercise at least 6 hours prior to testing. When the subjects initially arrived at the Exercise Physiology Laboratory basic anthropometric data, height, weight, and age were obtained. A Lunar Prodigy Dual Energy X-ray Absorptiometry (DEXA, General Electric Healthcare, Madison, WI) scan was performed to determine each participant's body composition. The DEXA machine was calibrated each day before use.

\subsubsection{Repetition Maximum}

Participants completed a general warm-up for 5 minutes on a treadmill. They then began the protocol for a 1 RM max test for the bench press exercise. Following the general warm-up they completed one set of 10 repetitions at $50 \%$ of their perceived $1 \mathrm{RM}$. The weight was then increased to $70 \%$ of their perceived 1 RM for 8 repetitions. Weight was increased again to $90 \%$ perceived $1 \mathrm{RM}$ for 3 to 5 repetitions. Weight was then incrementally increased $5 \%$ until the participant could not perform one repetition. Participants then followed the same protocol for determining 1 RM for the bent barbell row, and military press exercise. On a separate day (separated by 48 hours), participants completed the same protocol for determining one repetition maximum for the squat and deadlift exercises.

\subsubsection{Experimental Sessions}

Participants performed all experimental sessions at the same time of day to account for circadian variations in hormone levels. Upper Body Moderate Intensity: Participants completed a general warm-up for 5 minutes on a treadmill at $0.080 \mathrm{~km} / \mathrm{min}(3 \mathrm{mph})$. Participants then performed 2 sets of 9 repetitions at $70 \% 1 \mathrm{RM}$ for the bench press, bent barbell row, and military press exercises according to American College of Sports Medicine guidelines (Pescatello et al., 2014). One and a half minutes of rest were given between each set. Total work (J) was controlled for by the following equation:

$$
\mathrm{J}=\text { sets } \mathrm{x} \text { repetitions } \mathrm{x} \text { total amount of weight lifted (Gotshalk et al., 1997) }
$$

High Intensity Upper Body: Participants completed a general warm-up for 5 minutes on a treadmill at $0.080 \mathrm{~km} / \mathrm{min}$ ( $3 \mathrm{mph}$ ). They then performed a high intensity resistance training protocol consisting of 5 sets of 3 repetitions at 90\% 1 RM for the bench press, bent barbell row, and military press exercises. Three minutes of rest were given between each set. Moderate Intensity Lower Body: Participants completed a general warm-up for 5 minutes on a treadmill at $0.080 \mathrm{~km} / \mathrm{min}(3 \mathrm{mph})$. They then performed 2 sets of 9 repetitions at $70 \% 1 \mathrm{RM}$ for the squat and deadlift exercises. One and a half minutes of rest were given between each set. High Intensity Lower Body: Participants completed a general warm-up for 5 minutes on a treadmill at $0.080 \mathrm{~km} / \mathrm{min}(3 \mathrm{mph})$. They then performed 5 sets of 3 repetitions at $90 \% 1$ RM for the squat and deadlift exercises. Three minutes of rest were given between each set.

\subsubsection{Total Training Volume}

In order to keep training volume constant for each participant throughout each workout the number of sets and repetitions were adjusted accordingly. Some participants needed to complete more or less sets and repetitions based on their total weight lifted. Total work was held constant between each workout, both high intensity and moderate intensity as well as between the upper and lower body training sessions.

\subsubsection{Blood Sample Collection}

Blood samples (600 micro liters) were taken from the participant via finger stick using aseptic technique into an anticoagulant tube (Multivette $600 \mathrm{LH}$, Sarstedt, Fisher Scientific, Pittsburgh, PA). Samples were taken preexercise, post-exercise, and 60-minutes post-exercise. Samples were stored on ice for no more than $1 \mathrm{~h}$ before centrifugation at $3000 \mathrm{rpm}$ for 5-min (model 5415D, Eppendorf, Hamburg, Germany) and the plasma was stored at 80 degrees $\mathrm{C}$ (Revco, Thermo Scientific, Waltham, MA) prior to being analyzed. Testosterone was measured from each sample in duplicate using a commercially available AccuBind ELISA kit (Lake Forest, CA). The absorbance was read at $450 \mathrm{~nm}$ using a reference wavelength of $620-630 \mathrm{~nm}$ in a microplate reader (Epoch, BioTek, Winooski, VT). 


\subsection{Statistical Analyses}

A 2 (intensity: moderate, high) x 2 (muscle group: upper body, lower body) x 3 (time: pre, immediate post, 60-min post) ANOVA with repeated measures was used to analyze the data. Post hoc analyses were performed using paired t-tests when appropriate. The significance level was set at $\alpha=0.05$. All statistical analyses were calculated using SPSS Version 20 (IBM Corporation, Armonk, NY).

\section{Results}

\subsection{Time Comparison}

Testosterone concentration increased significantly (29.4\%) immediately following the upper body moderate intensity protocol $(\mathrm{F}=5.932, \mathrm{p}=0.026)$ compared to resting values and returned to near baseline by $1 \mathrm{~h}$ post exercise (see figure 1). Similarly, testosterone concentration obtained immediately following the lower body moderate intensity protocol was statistically higher $(27.1 \%, \mathrm{~F}=4.963, \mathrm{p}=0.024$, see figure 1$)$ and values returned to near baseline levels by 60-minutes post exercise. With respect to the upper body high intensity protocol, testosterone concentration was elevated $(15.2 \%)$ but not significantly different compared to rest $(\mathrm{F}=1.407, \mathrm{p}=$ 0.272). Similarly, testosterone concentration obtained immediately after the lower body high intensity exercise protocol was elevated $(17.5 \%)$ but not statistically significant $(\mathrm{F}=0.428, \mathrm{p}=0.658)$ compared to pre-exercise values.

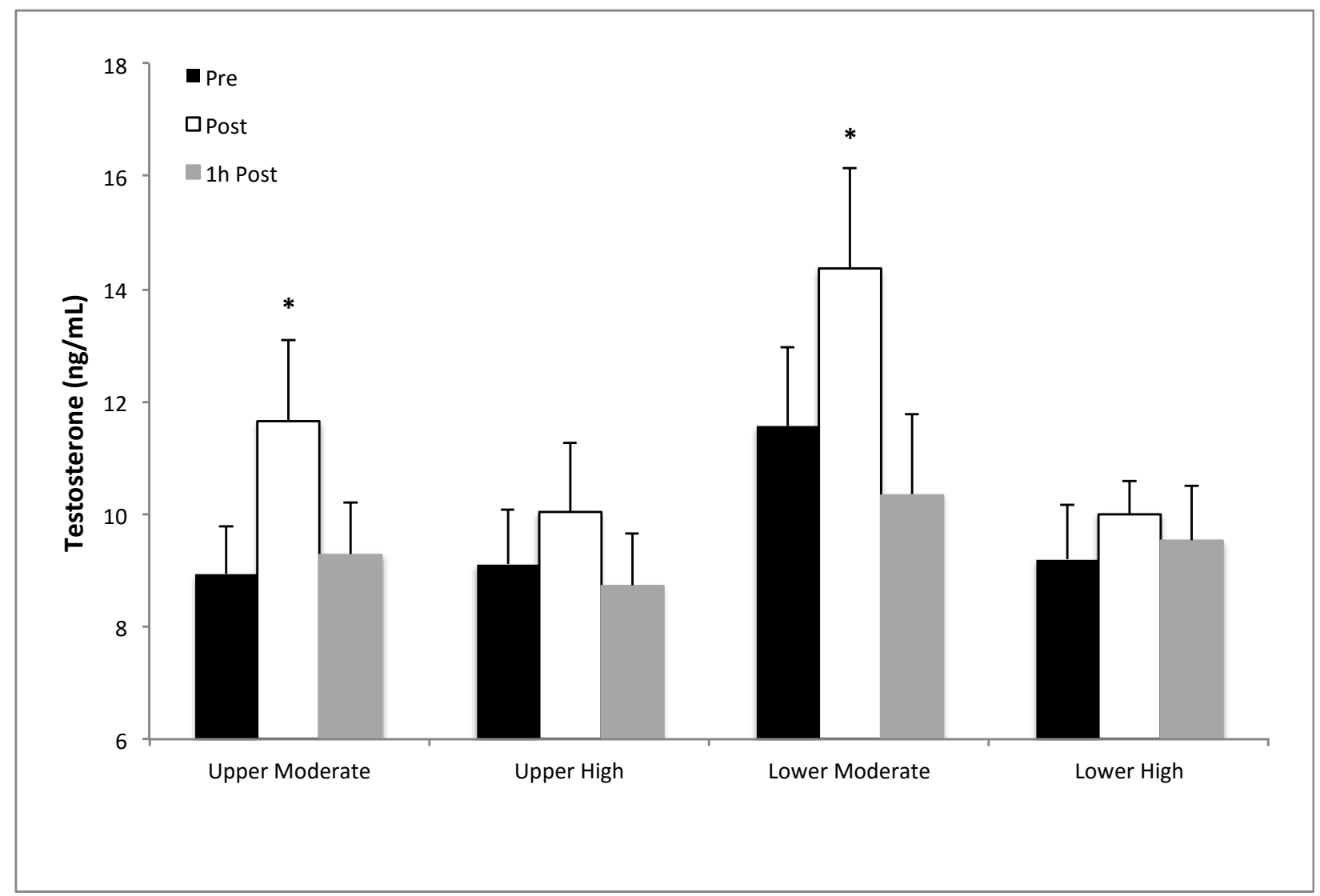

Figure 1. Testosterone response before (pre), immediately after (post) and $1 \mathrm{~h}$ after (60 post) in subjects $(\mathrm{N}=10)$ who completed acute resistance training of upper body moderate intensity (UM), upper body high intensity (UH), lower body moderate intensity (LM), and lower body high intensity protocols $(\mathrm{LH}) . *$ indicates significant increase compared to baseline levels $(\mathrm{p}<0.05)$. Error bars represent standard error.

\subsection{Upper Versus Lower Body Comparison}

An upper versus lower body comparison was performed for both moderate and high intensity protocols for postexercise testosterone values. With respect to the moderate intensity exercise protocol, post-workout testosterone concentrations were elevated following the lower body exercise session but not statistically different when compared to the upper body session ( $p=0.248$, see figure 2 ). Post exercise testosterone concentration was similar between upper and lower body high intensity exercise protocols $(\mathrm{p}=0.990)$ (see figure 2 ). 


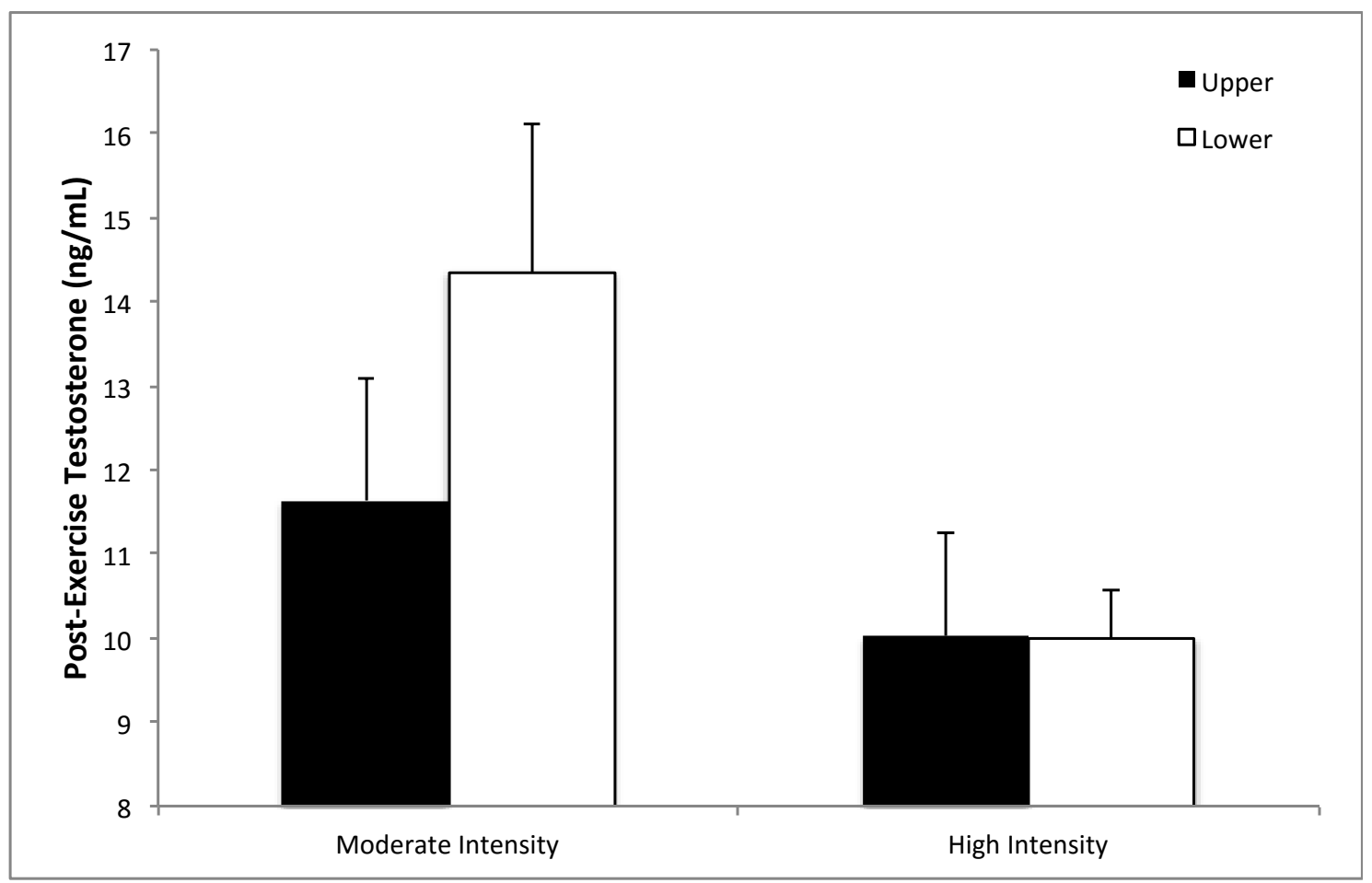

Figure 2. Immediate post-exercise testosterone response for upper versus lower body for both moderate and high intensity exercise protocols for subjects $(n=10)$.

\section{Discussion}

The increase in testosterone production following a bout of resistance exercise is related to the intensity of the exercise relative to the one repetition maximum weight and to the total volume of training. The purpose of this study was to determine the acute testosterone response to different intensities of resistance exercise when the total volume of work is the same. Total work for two intensities of one repetition maximum was equated by adjusting sets and repetitions for both upper and lower body exercises ( 2 sets of 9 repetitions at $70 \%$ one RM versus 5 sets of 3 repetitions at $90 \%$ one RM). We hypothesized that, with total work constant, both intensities of exercise would elicit an increase in testosterone, but a greater increase would occur following the high-intensity protocol. However, because the total work performed was the same for both upper-body and lower-body exercise at each intensity, testosterone should increase to the same extent.

Plasma testosterone concentration was significantly increased over baseline levels following the moderate intensity (70\% one RM) exercise as has been previously shown (Fry, Kraemer, \& Ramsey, 1998; Kraemer \& Ratamess, 2005; Migiano et al., 2010; Vingren et al., 2010), indicating that this intensity is sufficient to stimulate testosterone secretion. Interestingly, testosterone concentration was not significantly increased following the high intensity (90\% one RM) exercise protocol. These results contrast with previous findings that this intensity of resistance exercise was enough of a stimulus to elicit increases in testosterone production (Kraemer et al., 1998; Tremblay, Copeland, \& Van Helder, 2004). To equate total work between the moderate and high intensity protocols, the length of the sessions was shorter and the rest periods longer for the high intensity protocol, which may have dampened the testosterone response. This suggests a role for the total exercise duration in the testosterone response, since the total work performed was the same for both exercise protocols. Similarly, Gotshalk found that testosterone concentration was greater following a protocol of 3 sets of 10 repetitions compared with a protocol of 1 set of 10 repetitions (Gotshalk et al., 1997). Anecdotally, subjects in the present study subjectively felt that completing the moderate intensity protocol was the more difficult of the two protocols. Furthermore, while the total work performed has been shown to have an effect on post-exercise testosterone release (Gotshalk et al., 1997), the total training volume in this study was low compared with the training volume used by Gotshalk which resulted in a significant increase in testosterone following $90 \%$ one RM resistance exercise.

Performing the same total work in upper-body exercise as in lower-body exercise means a relative increase in intensity for muscles involved in upper body work, since the work is performed by a smaller muscle mass. This suggests a relatively greater feedback to the CNS from muscle receptors which should elicit a greater testosterone release. However, there were no significant differences in immediate post-exercise testosterone levels between upper- and lower-body exercise protocols for either moderate or high intensity sessions. This is in contrast to the results reported by Migiano et al. who found that 3 sets of 10 repetitions at an intensity near 70\% of one RM of upper-body exercise was not sufficient to increase testosterone (Migiano et al., 2010). In the present study, 2 sets of 10 repetitions utilizing three different upper-body exercises or two lower-body exercises was enough of a stimulus to induce a significant testosterone response immediately post-exercise. Furthermore, the magnitude of the response 
was similar between the muscles groups used, suggesting that if the total work performed is equal, lower-body exercise is not more effective than upper-body exercise in stimulating testosterone secretion. While the subjects in the current investigation were recreationally trained healthy individuals, it is tempting to speculate that these results may have implications for certain populations for whom lower-body exercise is not an option, such as those individuals who are wheelchair bound. It is possible that these individuals could still improve their fitness through upper-body resistance exercise, as long as the total volume of training is equal to that which would have potentially been accomplished by lower-body training.

\section{Conclusion}

This study provides evidence that a bout of moderate intensity $(70 \% 1 \mathrm{RM})$ resistance training for both the upper and lower body can significantly raise testosterone immediately following the bout. This confirms previous studies that demonstrated that a moderate intensity resistance-training session can affect the endocrine response. This information is useful for young, recreationally trained males because it provides evidence that moderate intensity (70\% 1RM) resistance training is sufficient for increasing testosterone immediately post-workout compared to high intensity (90\% 1RM) protocols that could be associated with a greater risk of injury in this population. Furthermore, moderate intensity protocols incorporating upper or lower body resistance exercise could be utilized to prevent tendencies toward intensity-related overtraining while still providing a beneficial hormonal response. Additionally, knowledge that an upper body moderate intensity resistance protocol significantly elevates testosterone could be important to spinal cord severed populations, such as paraplegics, in designing programs to maintain upper body muscle mass. While not an aim of the current investigation, further research should be conducted toward this potentially clinically important application.

\section{References}

Fahey, T. D., Rolph, R., Moungmee, P., Nagel, J., \& Mortara, S. (1976). Serum testosterone, body composition, and strength of young adults. Med Sci Sports, 8(1), 31-34. http://dx.doi.org/10.1249/00005768-197621000-00019

Faul, F., Erdfelder, E., Lang, A. G., \& Buchner, A. (2007). G*Power 3: a flexible statistical power analysis program for the social, behavioral, and biomedical sciences. Behav Res Methods, 39(2), $175-191$. http://dx.doi.org/10.3758/BF03193146

Fry, A. C., Kraemer, W. J., \& Ramsey, L. T. (1998). Pituitary-adrenal-gonadal responses to high-intensity resistance exercise overtraining. J Appl Physiol (1985), 85(6), 2352-2359.

Gotshalk, L. A., Loebel, C. C., Nindl, B. C., Putukian, M., Sebastianelli, W. J., Newton, R. U., . . Kraemer, W. J. (1997). Hormonal responses of multiset versus single-set heavy-resistance exercise protocols. Can J Appl Physiol, 22(3), 244-255. http://dx.doi.org/10.1139/h97-016

Hakkinen, K., Pakarinen, A., Newton, R. U., \& Kraemer, W. J. (1998). Acute hormone responses to heavy resistance lower and upper extremity exercise in young versus old men. Eur J Appl Physiol Occup Physiol, 77(4), 312-319. doi: 10.1007/s004210050339

Hough, J. P., Papacosta, E., Wraith, E., \& Gleeson, M. (2011). Plasma and salivary steroid hormone responses of men to high-intensity cycling and resistance exercise. J Strength Cond Res, 25(1), 23-31. doi: 10.1519/JSC.0b013e3181fef8e7

Kraemer, W. J., Hakkinen, K., Newton, R. U., McCormick, M., Nindl, B. C., Volek, J. S., . . Evans, W. J. (1998). Acute hormonal responses to heavy resistance exercise in younger and older men. Eur J Appl Physiol Occup Physiol, 77(3), 206-211. doi: 10.1007/s004210050323

Kraemer, W. J., \& Ratamess, N. A. (2005). Hormonal responses and adaptations to resistance exercise and training. Sports Med, 35(4), 339-361. http://dx.doi.org/10.2165/00007256-200535040-00004

Migiano, M. J., Vingren, J. L., Volek, J. S., Maresh, C. M., Fragala, M. S., Ho, J. Y., . . Kraemer, W. J. (2010). Endocrine response patterns to acute unilateral and bilateral resistance exercise in men. J Strength Cond Res, 24(1), 128-134. doi: 10.1519/JSC.0b013e3181a92dc5

Pescatello, Linda S., Arena, Ross, Riebe, Deborah, Thompson, Paul D., American College of Sports, Medicine, Lippincott, Williams, \& Wilkins. (2014). ACSM's guidelines for exercise testing and prescription. Philadelphia: Wolters Kluwer Health/Lippincott Williams \& Wilkins.

Tremblay, M. S., Copeland, J. L., \& Van Helder, W. (2004). Effect of training status and exercise mode on endogenous steroid hormones in men. J Appl Physiol (1985), 96(2), 531-539. doi: 10.1152/japplphysiol.00656.2003

Vingren, J. L., Kraemer, W. J., Ratamess, N. A., Anderson, J. M., Volek, J. S., \& Maresh, C. M. (2010). Testosterone physiology in resistance exercise and training: the up-stream regulatory elements. Sports Med, 40(12), 1037-1053. doi: 10.2165/11536910-000000000-00000

Weiss, L. W., Cureton, K. J., \& Thompson, F. N. (1983). Comparison of serum testosterone and androstenedione responses to weight lifting in men and women. Eur J Appl Physiol Occup Physiol, 50(3), 413-419. http://dx.doi.org/10.1007/BF00423247 